Title:

Lattice Coupling to Electronic and Magnetic Instabilities in High Magnetic Fields

Joe D. Thompson, MST-10

Thomas Graf, MST-10

Michael Hundley, MST-10

Alex Lacerda, Florida State University

Jon Lawrence, University of California at Irvine

John Neumeier, MST-10

Norman Phillips, University of California at Berkeley

OSTI

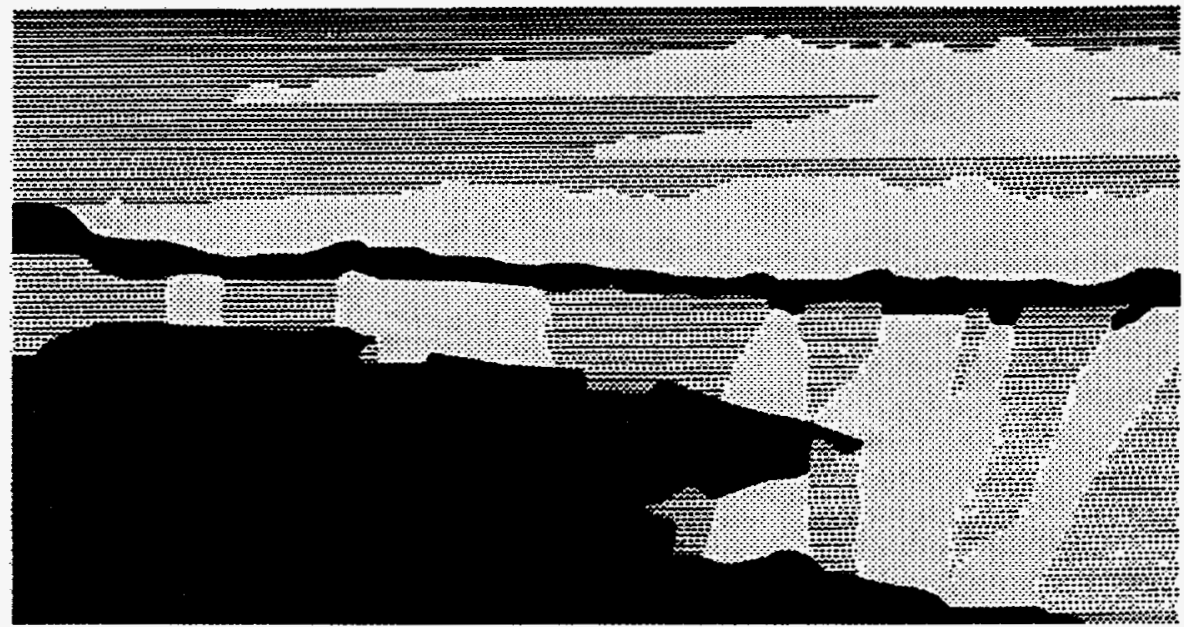

Los Alamos National Laboratory, an aftimattve action/equal opportunity employer, is operated by the University of Calliomia tor the U.S. Department of Energy under contract W-7405-ENG-36. By acceptance of this article, the publisher recognizes that the U.S. Government retains a nonexclusive, royaltyfree license to publish or reproduce the published form of this contribution, or to allow others to do so, for U.S. Government purposes. The Los Alamos National Laboratory requesis that the publisher identify this article as work performed under the auspices of the U.S. Department of Energy. 


\section{DISCLAIMER}

Portions of this document may be illegible in electronic image products. Images are produced from the best available original document. 


\title{
Lattice Coupling to Electronic and Magnetic Instabilities in High Magnetic Fields
}

\author{
Joe D. Thompson*, Thomas Graf, Michael Hundley, Alex Lacerda (Florida State \\ University/National High Magnetic Field Laboratory), Jon Lawrence (U.C. Irvine), \\ John Neumeier, and Norman Phillips (U.C. Berkeley)
}

\begin{abstract}
This is the final report of a three-year, Laboratory-Directed Research and Development (LDRD) project at the Los Alamos National Laboratory (LANL). The project focused on understanding electronic and magnetic instabilities in broad classes of materials in which the instabilities are coupled to the underlying crystallographic structure. Explaining these properties of materials poses outstanding theoretical and experimental challenges that are at the forefront of materials science/condensed matter physics. Very high magnetic fields available at the Los Alamos National High Magnetic Field Laboratory (NHMFL) are a key parameter in helping to provide this understanding. We have developed new experimental capabilities (thermal-expansion/magnetostriction, uniaxial stress and high-field heat capacity) needed to characterize how structure couples to the instabilities.
\end{abstract}

\section{Background and Research Objectives}

Properties of materials are determined ultimately by the configuration of their electronic states. Occasionally this configuration leads, for example, to enhanced mechanical characteristics or corrosion resistance that are desirable for applications. In other materials, it can produce unusual structural or electronic instabilities. Materials with these desirable or unusual properties are often labeled advanced materials, but quite frequently we do not understand fundamentally how these properties arise. It is important to provide this understanding for their desired properties to be realized fully and to guide the development of new advanced materials.

This project has focused on understanding classes of materials with unusual structural and/or electronic (including magnetic) properties. Quite generally, the properties can be attributed to instabilities that arise from the interplay between electronic configurations and the

* Principal investigator, e-mail: thompson_joe_david@lanl.gov 
underlying structural lattice and allow scientifically as well as technologically important ground states, e.g. high temperature superconductivity. In addition, this interplay is at the heart of a vast range of materials phenomena found in actinides or their compounds. Magnetic field, along with pressure and temperature, are the three fundamental thermodynamic variables that can be used both to discover these phenomena and to provide insight into their origin. Of these variables magnetic field is, within the U.S., the most underutilized but has the potential for discovering new phenomena and for providing a fundamental understanding of advanced materials.

Recently, a part of the new National High Magnetic Field Laboratory (NHMFL) was established at Los Alamos. The mission of this laboratory is to develop a world-class high field facility with emphasis on pulsed magnetic fields to $60 \mathrm{~T}$. It is important for the future of the NHMFL that a strong in-house research program be developed that utilizes these high-field resources. This need, combined with the importance of studying advanced materials at high magnetic fields, represents an outstanding opportunity. For this opportunity to be realized, a set of experimental techniques must be developed and applied to study novel materials as well as known materials exhibiting novel phenomena. We have developed two particularly important experimental capabilities to this end: high-field specific heat and high-field thermal expansion/magnetostriction. These two thermodynamic qualities are related to each other by the so-called Gruneisen parameter that provides a measure of lattice coupling to electronic and magnetic interactions. This lattice coupling is poorly understood, and is a research area that is virtually unexplored, but yet is fundamental to understanding strong electronic correlations that characterize broad classes of materials. Specific materials in which we believe these effects can be most clearly studied have been selected from examples of current theoretical and experimental interest. From this research we have (1) improved our understanding of materials and (2) brought new experimental capabilities that open new fields of investigation.

\section{Importance to LANL's Science and Technology Base and National R\&D Needs}

Los Alamos has a recognized strong experimental and theoretical competency in nuclear and advanced materials; prominent among these materials are actinides, high temperature superconductors and systems that exhibit heavy-fermion behavior. Common to all of these are electronic and magnetic instabilities that are coupled to the lattice and dominate their lowtemperature properties. Explaining these properties at a fundamental level poses theoretical and experimental challenges at the forefront of materials science/condensed matter physics. It is precisely these sorts of challenges that must be met if Los Alamos is to maintain and strengthen 
its core competency in nuclear and advanced materials. Although we have focused on specific materials as representative of properties to be explained, understanding these examples has scientific and technological implications beyond the limited scope of these investigations because we are addressing concepts and methodology as well as specific materials properties. In the process of achieving our objectives we also have (1) developed new materials diagnostics that will make the Los Alamos NHMFL unique among high field facilities in the U.S., (2) supported the substantial theoretical and experimental programs aimed at understanding advanced materials, and (3) enhanced international competitiveness in the area of high field research.

\section{Scientific Approach and Results to Date}

Our approach has been (1) to develop new characterization techniques needed to study lattice coupling to electronic and magnetic instabilities, particularly at high magnetic fields and (2) to apply these and existing techniques to examine how the interplay among structural, magnetic and electronic interactions produces the instabilities. In general, the interplay among interactions leads to a modification of the Fermi surface, through its renormalization by manybody (strong correlation) effects, or to an instability in the Fermi surface as evidenced by the opening of a gap over part or all of the surface. An outstanding example of the first case is heavy-fermion compounds in which many-body interactions renormalize the Fermi energy downward by two to three orders of magnitude. In this case, the characteristic energy scale of the correlated electrons becomes small, on the order of tens of Kelvin or equivalently tens of Tesla. The second case of Fermi surface instability represents cooperative phase transitions, such as superconductivity, long-range magnetic order, metamagnetic transitions and spin- or charge-density wave states. The energy scale in both cases is the range of magnetic fields available at the NHMFL. By studying the response of these materials at low temperatures to fields of this order, we can probe interactions responsible for their electronic and magnetic instabilities. These effects are known to produce either a significant change in the lowtemperature specific heat or a specific heat anomaly at the phase transition. From purely thermodynamic arguments, there must be a corresponding change in thermal expansion that measures the lattice coupling. Therefore, to study these effects, we have developed the ability to perform specific heat and thermal expansion measurements in high magnetic fields and at low temperatures.

Much has been accomplished. Most notable is that the capacitance dilatometry cell developed for thermal expansion measurements has been fully calibrated as a function of temperature and field and has been used to make the first magnetostriction (isothermal, field- 
induced length change) measurements at Los Alamos. (This is the only such capability in the U.S., to our knowledge.) We have measured the diagonal and off-diagonal components of the magnetostriction tensor of the electronically correlated small-gap semiconductor $\mathrm{Ce}_{3} \mathrm{Bi} 4 \mathrm{Pt}_{3}$. Although each tensor component is relatively large, the volume magnetostriction (a linear combination of the components) is relatively small, $\lambda_{\mathrm{V}}=(\mathrm{V}(\mathrm{H})-\mathrm{V}(0)) / \mathrm{V}(0)=2.75 \times 10^{-6}$ in $10 \mathrm{~T}$ at $4 \mathrm{~K}$, and comparable to that of strongly mixed valent metals, such as CeSn3 and CePd3. The coefficient of magnetostriction $S_{V}=\partial \lambda_{V} / \partial H^{2}$ exhibits a maximum near $50 \mathrm{~K}$, as does the thermal expansion and specific heat. Combining our measurements of magnetostriction, thermal expansion, specific heat, magnetic susceptibility and pressuredependent resistivity, we can show that the electronic free energy obeys a single scaling relationship, but that the electronic and magnetic Gruneisen parameters are inequivalent. This result supports the theoretical prediction that the charge and spin gaps in $\mathrm{Ce}_{3} \mathrm{Bi}_{4} \mathrm{Pt} 3$ are inequivalent. We also find that the field-dependent volume change cannot account for the large negative magnetoresistance in this material.

We have made an extensive set of high-field specific heat, resistivity and magnetization measurements on the strongly correlated metal $\mathrm{YbAgCu}_{4}$ and compared these to theoretical predictions of the Anderson impurity model. This model accounts well for the temperature and field dependence of the measured properties but the characteristic energy scales determined from the various measurements differ by nearly a factor of two, which is not expected from the model. This discrepancy is not understood but could arise from a field-dependent $4 f$ occupancy (valence), which is not included in the model but could be tested experimentally by field-dependent inelastic neutron scattering experiments. Our work is the first serious test of the Anderson model using magnetic field as the primary variable and the first to suggest the possibility of a field-dependent valence in electronically correlated metals.

Finally, we have used very high field magnetoresistance measurements to study properties of $\mathrm{Sr}$-doped $\mathrm{La}_{2} \mathrm{CuO}$. In the case of $\mathrm{La}_{1.99} .9 \mathrm{Sr} .01 \mathrm{CuO}_{4}$, we have mapped the field-temperature phase diagram which can be understood if there is strong coupling between doped charge carriers and interlayer order of the out-of-plane $\mathrm{Cu}^{2+}$ spin component. Very careful measurements of longitudinal and transverse magnetoresistance of La1.925 $\mathrm{Sr} .075 \mathrm{CuO}_{4}$ show that, contrary to previous reports, the magnetoresistance follows Kohler's rule and we explain these results as arising from eccentric hole-pockets on the Fermi surface, an interpretation consistent with band structure calculations.

During the past three years, we have developed new experimental capabilities of high field specific heat, state-of-the-art capacitance dilatometry (thermal expansion and magnetostriction) and uniaxial stress (to $4 \mathrm{kbar}$ ). These capabilities are now available to the NHMFL, as originally proposed. We have used these capabilities to study a wide range of 
advanced materials in which electronic and magnetic instabilities are coupled to the underlying lattice and have enhanced substantially our understanding of these effects.

\title{
Publications
}

[1] Hundley, M.F.; Neumeier, J.J.; Thompson, J.D.; Lacerda, A.; and Canfield, P.D, "Magnetostriction and Thermal Expansion of the Kondo Semiconductor $\mathrm{Ce}_{3} \mathrm{Bi}_{4} \mathrm{Pt} 3$,"

Physical Phenomena in High Magnetic Fields-II, to be published (1995).

[2] Lacerda, A.; Graf, T.; Hundley, M.F.; Torikachvili, M.S.; Lawrence, J.M.; Thompson, J.D.; Gajewski, D.; Canfield, P.C.; and Fisk, Z., "High Field Magnetotransport and Specific Heat in YbAgCu4," Physica B, 206\&207, 358 (1995).

[3] Graf, T.; Lawrence, J.M.; Hundley, M.F.; Thompson, J.D.; Lacerda, A.; Haanappel, E.; Torkachvili, M.; Fisk, Z.; and Canfield, P.C., "Resistivity Magnetization and Specific Heat of YbAgCu4 in High Magnetic Fields," Phys. Rev. B, 51, 15053 (1994).

[4] Lacerda, A.; Graf, G.; Cho, J.H.; Thompson, J.D.; and Maley, M.P., "High Field Magnetoresistance of La1.99Sr.01CuO4+d," Physica C, 235-240, 1353 (1994).

[5] Lacerda, A.; Rodgriguez, J.P.; Hundley, M.F.; Fisk, Z.; Canfield, P.C.; Thompson, J. D.; and Cheong, S-W., "Magnetoresistance in the Normal State of La1.925Sr.075CuO4+d," Phys. Rev. B, 49, 9097 (1994).

\section{DISCLAIMER}

\begin{abstract}
This report was prepared as an account of work sponsored by an agency of the United States Government. Neither the United States Government nor any agency thereof, nor any of their employees, makes any warranty, express or implied, or assumes any legal liability or responsibility for the accuracy, completeness, or usefulness of any information, apparatus, product, or process disclosed, or represents that its use would not infringe privately owned rights. Reference herein to any specific commercial product; process, or service by trade name, trademark, manufacturer, or otherwise does not necessarily constitute or imply its endorsement, recommendation, or favoring by the United States Government or any agency thereof. The views and opinions of authors expressed herein do not necessarily state or reflect those of the United States Government or any agency thereof.
\end{abstract}

